Published in final edited form as:

Curr Protoc Immunol. ; 104: Unit-15.25.. doi:10.1002/0471142735.im1525s104.

\title{
Dextran Sulfate Sodium (DSS)-Induced Colitis in Mice
}

\author{
Benoit Chassaing ${ }^{1}$, Jesse D. Aitken ${ }^{1}$, Madhu Malleshappa ${ }^{1}$, and Matam Vijay-Kumar ${ }^{2,3}$ \\ ${ }^{1}$ Center for Inflammation, Immunity, \& Infection, Department of Biology, Georgia State University, \\ Atlanta, Georgia \\ ${ }^{2}$ Department of Nutritional Sciences, The Pennsylvania State University, University Park, \\ Pennsylvania \\ ${ }^{3}$ Department of Medicine, The Pennsylvania State University College of Medicine, Hershey, \\ Pennsylvania
}

\begin{abstract}
Inflammatory bowel diseases (IBD) mainly comprised of Ulcerative Colitis and Crohn's Disease are complex and multifactorial disease with unknown etiology. For the past 20 years, to study human IBD mechanistically, number of murine models of colitis has been developed. These models are indispensable tools to decipher underlying mechanisms of IBD pathogenesis as well as to evaluate number potential therapeutics. Among various chemical induced colitis models, DSSinduced colitis model is widely used because of its simplicity and many similarities with human ulcerative colitis. This model has both advantages and disadvantages that need to be considered when employed. The current protocol aimed to extensively describe the DSS-induced colitis model, focusing on its detailed protocol as well as factors that could affect DSS-induced pathology.
\end{abstract}

\section{INTRODUCTION}

The mammalian gastrointestinal tract is continuously exposed to numerous bacteria as well as food-derived and environmental toxins and, as such, is highly vulnerable to disease. Two major inflammatory bowel diseases (IBD) of the gastrointestinal tract, Crohn's Disease (CD) and Ulcerative Colitis (UC), are characterized by both acute and chronic inflammation of the intestine with multifactorial etiology (Xavier and Podolsky, 2007). In the last few decades, numerous animal models have been developed to characterize the complexity of IBD pathogenesis, delineating underlying molecular mechanisms and evaluating potential human therapeutics (Chassaing and Darfeuille-Michaud, 2011).

Perhaps the most widely used mouse model of colitis employs dextran sodium sulfate (DSS), a chemical colitogen with anticoagulant properties, to induce disease. DSS is a water-soluble, negatively charged sulfated polysaccharide with a highly variable molecular weight ranging from 5 to $1400 \mathrm{kDa}$. The most severe murine colitis, which most closely resembles human UC (Okayasu et al., 1990), results from administration of 40-50kDa DSS in drinking water. The mechanism by which DSS induces intestinal inflammation is unclear but is likely the result of damage to the epithelial monolayer lining the large intestine allowing the dissemination of proinflammatory intestinal contents (e.g. bacteria and their products) into underlying tissue. The DSS colitis model is very popular in IBD research due to its rapidity, simplicity, reproducibility and controllability. Acute, chronic and relapsing models of intestinal inflammation can be achieved by modifying the concentration of DSS and the frequency of administration. 
An important caveat pertaining to DSS colitis: unlike in human disease, T and B cells are not required for development of colitis. This apparent limitation, however, allows for the study of colitis in both SCID and RAG-KO mice (both characterized by the absence of adaptive immunity). Hence, the acute DSS colitis model is particularly useful when studying the contribution of the innate immune system to the development of intestinal inflammation. In addition, intestinal bacteria are essential for the development of robust colitis. The Basic Protocol in this Unit discusses the induction of DSS colitis as well as methods to monitor disease activity, prepare pertinent RNA, fecal and serum samples and measure epithelial barrier function, cell proliferation and migration.

\section{BASIC PROTOCOL}

\section{Induction and evaluation of DSS colitis in mice}

The following protocol describes the induction of colitis in mice via administration of DSS. In light of the indispensability of the microbiota in this model of colitis, all groups of mice should share a common origin (strain, vendor and colony). Specifically, when studying colitis susceptibility in wild type and genetically deficient and/or transgenic mice, use of littermates is highly recommended.

Upon addition of DSS to drinking water, mice should be monitored daily for body weight and presence of blood in the stool. Initially, there may be a slight increase in body weight (day 3-4) depending on the dose of DSS and strain of mouse. It may be useful, especially for those unfamiliar with the model, to monitor the onset of disease by measuring non-invasive fecal markers of inflammation. Generally, and in accordance with IACUC recommendations, loss of $25-30 \%$ of initial body weight should be considered as death with euthanasia administered according to institutional guidelines. As disease severity and mortality can be variable, a sufficient number of animals (matched for age, gender and body weight) should be used, preferably 5-10 mice per treatment group. Controls should be given unsupplemented water.

\section{Materials}

- Dextran Sulfate Sodium (DSS) salt, Reagent Grade (MP Biomedicals (formerly ICN Biomedicals) M.W 36-50kDa, Ref $=160110$ ), 2.0-5.0\% in autoclaved drinking water.

- Male or female mice, preferably 8 weeks old. C57BL/6J and BALB/c background mice are recommended due to their prevalence in existing literature.

Note: Since the colitogenic potential of DSS varies between vendors and even manufacturing lots, purchasing DSS in bulk $(500 \mathrm{~g})$ and determining the optimal concentration required to induce robust colitis over a period of 7 days in the mouse strain of choice is recommended. Initially, administer a range of doses between $1.5 \%$ and $3.0 \%$ for BL6 and 2.5-5.0\% for BALB/c mice and monitor colitis development by measuring body weight, occult blood and biomarkers of gut inflammation in the feces. This initial experiment will allow the selection of a DSS dose at which colitis development occurs in a slow and steady fashion in order to ensure consistency and reproducibility in later studies.

- Fecal occult blood: Hemoccult Dispensapak Plus from Beckman Coulter (Ref = 61130).

- Serum separator tubes: BD Microtainer tubes $(\operatorname{Ref}=365956)$.

- 4 and 40Kda FITC-dextran (Sigma, Ref = FD4) to measure epithelial barrier permeability. 
- Brominated deoxyuridine (BrdU: Invitrogen, Ref $=$ E10187) to ascertain epithelial cell proliferation and migration.

Additional reagents and equipment for administering inhaled anesthesia to mice, fixing tissue in 10\% PBS-buffered formalin, hematoxylin and eosin (H\&E) staining, immunohistochemistry, RNA extraction, myeloperoxidase assay and fecal biomarker assay are described elsewhere.

\section{Preparation of mice for DSS treatment}

1. On the day of DSS administration (day 0), labeled (using ear punch or any other convenient method) groups of control and experimental mice should be weighed and, if required, average group weight equilibrated so as to eliminate any significant weight difference between groups. Labeling individual mice aids in tracking their susceptibility to DSS-induced pathology. Should it be necessary to equilibrate average group body weights, minimize mixing mice over 4 weeks old between different cages, especially males, as they may injure each other by fighting. If possible, equilibrate group body weights before this point and house the mice until they reach the desired age. Stool collected prior to DSS administration can be used as a control when assaying fecal markers of inflammation. If you are testing a compound for its anti-colitogenic properties, determine the route, dose, volume, vehicle and mode (prophylactic or therapeutic) of administration. If the drug is water insoluble, the appropriate vehicle should be given to the control group in a similar fashion. Limit the volume administered to $0.2 \mathrm{ml}$ (i.p., i.g., i.v. or s.c.). Prepare an optimized concentration of DSS in autoclaved water by weighing DSS powder and mixing until a clear solution is achieved. Fill the cage water bottle with $100 \mathrm{ml}$ of DSS water, an amount generally sufficient for 5 mice for 2-3 days. Control mice should get the same amount of water without DSS.

Note: Observe water intake in each group of mice by measuring leftover water. This measurement is necessary as some genetically modified mice with hypermetabolic activity (e.g. Stearoyl CoA Desaturase (SCD-1) deficient mice (Macdonald et al., 2009)) may consume more DSS water than their controls and thereby develop relatively more severe colitis. Alternatively, different therapeutic strategies or other aspects of the experiment may affect food intake and thus lower consumption of DSS water. While DSS is quite stable at room temperature, DSS water should be changed every 2 days or if any growth (turbidity) in the bottles is observed. The optimal DSS concentration may vary due to any number of the aforementioned factors and should be determined by individual laboratories.

2. Body weight should be measured daily, as should the presence of occult blood. The following scoring system can be employed for the comparative analysis of intestinal bleeding: Normal stool consistency with negative hemoccult: 0; Soft stools with positive hemoccult: 1; Very soft stools with traces of blood: 2; Watery stools with visible rectal bleeding: 3 . Stool reserved for the measurement of inflammatory markers should be frozen at $-20^{\circ} \mathrm{C}$.

Collection of feces: Collect feces by placing a single mouse in an empty cage without bedding material for 15-30 min. As DSS administration continues and inflammation becomes more robust, the time required for collection will increase. With sterile forceps, collect feces in a microfuge tube and use one pellet to monitor occult blood. 
3. Body weights may slightly increase during the first three days and begin to decrease gradually with the initiation of bleeding. There is no hard and fast rule that DSS should be given for 7 days, rather it is up to the investigator to determine when mice should be sacrificed based on significant body weight loss and bloody diarrhea. For instance, MyD88 deficient mice exhibit DSS induced pathology much earlier than WT mice (Araki et al., 2005).

4. Direct visualization of DSS-induced colonic mucosal damage in vivo can be performed using the Coloview system (Karl Storz Veterinary Endoscopy, Tuttlingen, Germany, Figure 1B). Under 1.5-2.0\% isoflurane anesthesia, $3 \mathrm{~cm}$ of the proximal colon can be visualized following inflation of the colon with air. Endoscopic damage can be scored by assessing the following: colon translucency (0-3), presence of fibrin attached to the bowel wall (0-3), granular aspect of the mucosa (0-3), morphology of the vascular pattern (0-3) stool characteristic (normal to diarrhea; $0-3$ ) and presence of blood in the lumen (0-3), generating a maximum score of 18 .

5. Four and 24 hours before sacrifice, mice should be given BrdU intraperitoneally as recommended by the supplier (Invitrogen) to monitor intestinal epithelial cell proliferation and migration by specifically staining BrdU histologically.

6. On the day of sacrifice, mice should be deprived of food for $4 \mathrm{~h}$, given FITCdextran tracer ( 4 or $40 \mathrm{KDa}, 0.6 \mathrm{mg} / \mathrm{g}$ body weight) intragastrically in $0.1 \mathrm{ml} \mathrm{PBS}$ and bled (using convenient method) after $3 \mathrm{~h}$ to collect hemolysis-free serum. Intestinal permeability correlates with fluorescence intensity of appropriately diluted serum (excitation, 488 nm; emission, $520 \mathrm{~nm}$; Hitachi F-4500 Fluorescence Spectrophotometer). Prepare a standard curve for FITC-dextran by serially diluting a known amount of FITC-dextran in mouse serum.

Note: If well-planned, steps 5 and 6 can be performed on the same groups of mice.

7. On the day of termination, perform terminal bleeding and euthanize mice in accordance with approved institutional animal ethical protocols.

Note: To generate a quantitative measurement of intestinal inflammation, serum keratinocyte derived chemokine (KC) and/or lipocalin 2, which correlates with disease activity, may be measured via Duoset ELISA kits (R \& D Systems) following manufacturer recommendations. For this purpose, dilute control serum samples 1:2 or 1:4 for KC and 1:200 for lipocalin 2. Samples from DSS-treated mice require a much higher dilution.

8. Spray $70 \%$ ethanol and carefully open the mouse by ventral midline incision. Remove and weigh the spleen. Increased spleen weights generally correlate with the extent of inflammation and anemia. Further, plating spleen lysates $(100 \mathrm{mg} / \mathrm{ml}$ in PBS) on non-selective agar plates also indicates the extent of disseminated intestinal bacteria and should correlate with FITC-dextran permeability data.

9. Before collecting the colon, isolate mesenteric lymph nodes if planning to study their enlargement, characterize immune cell populations and/or assay gut bacteria translocation correlating with DSS-induced pathology.

10. Lift the colon with forceps and carefully pull until the cecum is visible. Dissecting colons from severely inflamed DSS-treated animals is tricky as this tissue thins, shortens and becomes attached to extraintestinal tissues. 
11. Isolate the colon and cecum by separating them from the small intestine at the ileocecal junction and from the anus at the distal end of rectum. At this time, gross pictures of intestine from all groups of mice or one representative from each group (as shown in Figure 1A), from cecum to rectum, can be taken.

12. To measure length, straighten but do not stretch the colon. The colon can then be separated from the cecum (again, at the ileocecal junction) and quickly flushed (5-10 ml syringe with feeding needle (18G-3" Straight $2.25 \mathrm{~mm}$ ball, Braintree Scientific Inc) using cold PBS to remove feces and blood. The cecum can be discarded as little or no inflammation is induced by DSS in this region.

Note: After flushing with PBS, colon weights can be taken. In accordance with observed tissue wasting, severely inflammed colons exhibit reduced weight as both correlate with the severity of acute inflammation. In chronic models of colitis, unlike acute DSS-induced colitis, inflammation is associated with increased colon weight due to the granulomatic nature of inflammation.

13. Cut colons into pieces depending on need. It is important to compare the same region of the colon from different groups of mice when performing any analysis. An example would be to use the proximal colon for MPO (frozen immediately), the middle portion for RNA isolation stored in RNAlater) and the rectal region for histology (fixed in $10 \%$ formalin in a cassette).

14. For colonic Myeloperoxidase (MPO) assay, weigh the tissue and freeze $\left(-80^{\circ} \mathrm{C}\right)$ until analysis. Colonic MPO is a marker for neutrophils, and its concentration in tissue correlates with the extent of neutrophil infiltration.

a. Weigh colonic tissue (50-100 mg) and wash thoroughly in PBS until free of fecal matter and store at $-80^{\circ} \mathrm{C}$ until analyzed.

b. Homogenize tissue in $0.5 \%$ hexadecyltrimethylammonium bromide (Sigma, Ref = H6269) in 50 mM PBS, pH 6.0.

c. Freeze-thaw 3 times, sonicate for $10 \mathrm{sec}$ to get a homogenous tissue suspension and centrifuge at high speed at $4^{\circ} \mathrm{C}$.

d. Assay MPO in the clear supernatant in a 96 well plate by adding $1 \mathrm{mg} / \mathrm{mL}$ of dianisidine dihydrochloride (Sigma, Ref $=\mathrm{D} 3252$ ) and $0.5 \times 10^{-4} \%$ $\mathrm{H}_{2} \mathrm{O}_{2}$, measure optical density at $450 \mathrm{~nm}$. Human neutrophil MPO (Sigma, Ref = M6908) can be used as a standard (Range: 0.5-0.015 U/ml). One unit of MPO activity is defined as the amount needed to degrade 1.0 $\mu \mathrm{mol}$ of peroxide $/ \mathrm{min}$ at $25^{\circ} \mathrm{C}$ [24].

Note: A brownish yellow color should develop slowly over a period of 10-20 min. If color development is too rapid, particularly in DSS-treated colonic samples, dilute and repeat the assay. Express MPO as U/mg protein or U/g tissue.

15. For qRT-PCR analysis, place a piece of colon $(50 \mathrm{mg})$ in RNAlater (Sigma, Ref $=$ R0901) until RNA extraction. For longer storage, tissue in RNAlater should be frozen at $-20^{\circ} \mathrm{C}$. On the day of RNA extraction, remove colons from RNAlater and extract RNA by any standard procedure (e.g. Trizol method). As traces of DSS in colonic tissue have been known to interfere with PCR amplification, it is necessary to remove all polysaccharides, including DSS, via lithium chloride method as previously described (Chassaing et al., 2012). 
16. For histological staining (e.g. Hematoxylin \& Eosin (H\&E) or Periodic Acid-Schiff (PAS) staining for goblet cells), swiss rolls are recommended. This will allow observation of colonic pathology in extended regions compared to standard transverse or longitudinal sections. Cut each piece of colon longitudinally, wrap it around a toothpick wetted with PBS and place it in a cassette. Following 24 hours of fixation in $10 \%$ buffered formalin, transfer cassettes to $70 \%$ ethanol until analyzed. For special staining (e.g. immunohistochemistry, immunofluorescence) with specific antibodies, frozen sections fixed in the cryoprotective embedding medium, OCT (Optimal Cutting Temperature, Tissue-Tek, Ref = 4583), are recommended. Additionally, if studying mucin layers and bacterial adhesion/ translocation, abstain from flushing the colon with PBS and immerse in Carnoy's solution (Johansson et al., 2008) as this preserves mucus architecture. Formalinfixed colonic tissue can be stained for BrdU using BrdU antibodies (Invitrogen). Alternatively, Ki67 can be used to measure epithelial cell proliferation.

Histologic scoring: Blinded histologic scoring can be performed on $\mathrm{H} \& \mathrm{E}$ stained colonic tissue as follows. Each section is assigned four scores based on the degree of epithelial damage and inflammatory infiltration into the mucosa, submucosa and muscularis/serosa. Each of the four scores is multiplied by 1 if the change was focal, 2 if it was patchy and 3 if it was diffuse. The 4 individual scores per colon are added, resulting in a total scoring range of 0-36 per mouse. The average scores for control and DSS-treated groups can then be tabulated.

17. Culturing washed colons ex vivo yields key information regarding the severity of inflammation. Specifically, colons (approximately $1.0 \mathrm{~cm}$ ), cut longitudinally, should be washed thrice serially in HBSS with $1.0 \%$ antibiotics (penicillin \& streptomycin, Fisher, Ref = BP2959). Place washed colons in a well (24 well plate) containing $1.0 \mathrm{ml}$ of serum-free RPMI1640 medium with $1.0 \%$ antibiotics (penicillin \& streptomycin) and incubate at $37^{\circ} \mathrm{C}$ with $5.0 \% \mathrm{CO} 2$ for $24 \mathrm{~h}$. Collect supernatants and centrifuge for $10 \mathrm{~min}$ at $4^{\circ} \mathrm{C}$ and store at $-80^{\circ} \mathrm{C}$ until analysis for proinflammatory cytokines.

Note: Chronic colitis can be induced in mice by continuous treatment of mice with a low dose of DSS or via cyclical administration of DSS, for example 4 cycles of DSS treatment for 7 days followed by 7-10 days of water. Apart from this, all procedures remain the same.

\section{CRITICAL PARAMETERS AND TROUBLESHOOTING}

The successful and reproducible induction of DSS-induced colitis depends on numerous key factors, including DSS source, lot \#, molecular weight, concentration, duration, mouse strain, source, age, gender and body weight as well as environmental factors including the hygienic condition of the vivarium (Nell et al., 2010). If high mortality is observed, suggesting high susceptibility to DSS, a decreased dose of DSS should be adopted. If no or weak colitis is observed, suggesting low susceptibility, and increase in DSS concentration/ and or duration should be considered.

Mice

As in any other model of colitis, the genetic background of the animals employed is the most important determinant of the outcome of DSS-induced pathogenesis. Investigators should first determine the strain they are using and standardize the optimal DSS dosage and duration such that colitis develops in a slow and steady manner. The slow and steady onset 
of colitis is very critical in differentiating disease pathogenesis between different genetic backgrounds and therapeutic interventions.

We have also found that, generally, DSS-induced disease development occurs more gradually in young animals when compared to older mice. That younger mice exhibit relatively lower demand for food and, thus, reduced water intake, could be a potential reason for the delayed onset of DSS-induced gut inflammation. The optimal age for successful and easily reproducible induction of DSS colitis ranges between 6-8 weeks.

Both males and females develop DSS-induced colitis robustly, but males rapidly develop more significant and aggressive disease than females. Thus, the ability to induce disease as well as severity of the clinical course of DSS-induced colitis depends on many factors besides the genetic background of the animal employed.

When studying the therapeutic potential of a compound in ameliorating DSS-induced colitis, care should be taken when handling the mice and determining volume and vehicle. Daily administration of a drug i.p., i.v. or orally is not recommended and, as such practice results in severe and potentially confounding stress, alternative approaches should be considered.

Although it is difficult to measure DSS-induced colitis quantitatively, body weight measurement and feces collection followed by analysis of a biomarker of gut inflammation should be done. Recently, we have found that quantification of fecal lipocalin- 2 by ELISA is a useful approach as it is a non-invasive, sensitive and broadly dynamic biomarker of intestinal inflammation (Chassaing et al., 2012).

\section{Outside environmental factors}

As in other colitis models, the environment of the vivarium plays a critical role in the development of DSS-induced colitis. Specifically, under germ-free conditions, colitis development is mild. In contrast to this, our unpublished results indicate that WT C57BL6 mice from vendors, which have been strictly maintained in hygienic conditions, are more susceptible to developing severe colitis than WT C57BL6 maintained and bred in our institutional animal facility. One explanation for differences could be the presence of, or prior infection with, common animal facility pathogens such as Helicobacter, segmented filamentous bacteria, norovirus, pinworms, etc. Such exposure could also alter the innate immune response and thus significantly influence the development of DSS-induced colitis (Cerf-Bensussan and Gaboriau-Routhiau, 2010). To overcome this potential problem, it is always advisable to use mice from a specific vendor or to compare experimental groups with WT littermates harboring similar microbiota in order to obtain meaningful and reproducible results.

In general, DSS administration results in severe bleeding, diarrhea and sticky stools, which dirty the cages more quickly than normal. However, it is advisable to change the cages as infrequently as possible since murine coprophagy helps recycle key nutrients. Further, changing cages daily may result in increased food and water consumption and unnecessary stress. Should any cage require changing, the cages of all groups should be changed simultaneously.

DSS is a highly water-soluble compound and, when it is dissolved in water, yields a clear solution. The colitogenic potential of DSS depends on its molecular weight. Generally, a molecular weight of $36-50 \mathrm{Kda}$ is employed when inducing colitis. The use of lower molecular weight DSS (5Kda) may result in milder colitis while higher weight DSS (500Kda) does not cause colonic injury (Kitajima et al., 2000). Although DSS powder is 
quite stable at room temperature, it is best to prepare DSS water on the day of administration. DSS water should be thoroughly mixed with a magnetic stir bar and completely dissolved before administration. Undissolved salt may clog the water bottle's outlet or otherwise influence water intake, potentially contributing to erroneous results. Frequently checking for turbidity in the water bottle is recommended as turbidity indicates microbial growth and necessitates replacement with a new bottle containing freshly prepared DSS water. This is very important, especially during the induction of chronic colitis with multiple alternating cycles DSS-water and water administration. It is best practice to use autoclaved water when preparing DSS solutions; the same water should be given to control groups.

\section{Molecular and histological features of DSS-induced colitis}

DSS carries a highly negative charge contributed by sulfate groups, is toxic to the colonic epithelia and induces erosions that ultimately compromise barrier integrity resulting in increased colonic epithelial permeability (Figure 2). Further, its anticoagulant property aggravates intestinal bleeding. For unknown reasons, DSS-induced extensive pathology is confined to the large intestine, specifically the distal colon where an enormous number of microorganisms live. The mechanism by which DSS passes through mucosal epithelial cells remains unclear, but a recent publication suggests that DSS induces colitis in mice by forming nano-lipocomplexes with medium-chain-length fatty acids (MCFAs) in the colon (Laroui et al., 2012). The specificity of DSS to the colon could be a function of water and electrolyte absorption in the presence of numerous bacteria. Theoretically, negativelycharged DSS may damage negatively-charged (contributed by anionic membrane phospholipids) cell membranes by repulsive forces but the lack of DSS-induced inflammation with lower or higher molecular weights argues against this.

DSS administration induces clinical signs of disease as soon as 1 day post treatment, with changes in the expression of tight junction proteins (Poritz et al., 2007) and increased expression of proinflammatory cytokines (Yan et al., 2009). These modest initial effects are followed by increasingly drastic symptoms, including increased intestinal permeability, severe bleeding and, finally, mortality. The clinical and histological features induced by DSS mirror those seen in human IBD, particularly UC. Acute histological changes in the colon can be induced by relatively short exposure (4-7 days) to a high dose of DSS. Chronic (advanced) lesions can be induced by continuous treatment of low dose DSS or via cyclical DSS application. Acute histological changes are associated with clinical symptoms such as weight loss, diarrhea, bloody stools, hunched back and death.

Typical histological changes induced by DSS include mucin and goblet cell depletion, epithelial erosion and ulceration. Further, DSS induces an influx of neutrophils into the lamina propria and submucosa (Figure. 2). Transepithelial migration of neutrophils (cryptitis) and extensive migration of neutrophils through the mucosal epithelium and into the crypt lumen (crypt abcess) are commonly associated with human IBD but occasionally reported in DSS-induced pathology. Chronic DSS colitis results in mononuclear leukocyte infiltration, crypt architecture disarray and a widening of the gap between the base of the crypt and muscularis with deep mucosal lymphocytosis also being commonly associated. The transmural inflammation associated with TNBS colitis is absent in DSS colitis.

\section{Anticipated Results}

Mice with high susceptibility to DSS show severe rectal bleeding and weight loss within 3-4 days of DSS administration. Mice will exhibit visible signs of illness, including a hunched back, raised fur, symptoms of sepsis and reduced mobility as a result of diarrhea and anemia. Usually, mice on DSS will lose $20-30 \%$ of body weight depending on the 
concentration and duration of DSS. Graphical representation of \% loss/gain in body weight is a standard way to represent disease progression in this model of colitis. Body weight loss and rectal bleeding are also associated with thinning and shortening of the colon and enlargement of mesenteric lymph nodes and spleen (Figure 1A). Concomitantly, severe colitis will lead to superficial inflammation of the colon characterized by epithelial erosion and ulceration, crypt abscess, loss of goblet cells, loss of the mucus layer and substantial neutrophil infiltration into the lamina propria (Figure 1C) which can be summarized via graphical representation of histologic score or disease activity index. In chronic DSS colitis, proinflammatory immune cells (T cells, macrophages) outnumber neutrophils, the dominant effector cell type in acute colitis. Although frequent deaths in highly susceptible strains are common, mice may not develop robust disease due to any number of the aforementioned factors and/or genetic resistance.

\section{Time Considerations}

The increasingly widespread use of DSS-induced colitis by researchers is a function of this model's simplicity and reproducibility. In optimal conditions, disease induction occurs within 3-7 days following DSS administration. During this period, severe colonic bleeding occurs, mimicking the superficial inflammation seen in ulcerative colitis. As there are no special skills required for DSS administration, investigators can easily employ multiple groups to test disease susceptibility between genetic backgrounds or evaluate the efficacy of prophylactic or therapeutic strategies. For experienced laboratory personnel, the analysis of DSS-induced colitis can be completed in 2 weeks including evaluation of feces, serum, and colon culture supernatants for biomarkers and proinflammatory cytokine expression at both the mRNA and protein levels as well as histologic examination and specialized staining procedures.

\section{COMMENTARY}

\section{(I) Background Information: murine models of intestinal inflammation}

Over the last 15-20 years, the development of small animal (rodent/murine) models of intestinal inflammation has facilitated significant advances in our understanding of human inflammatory bowel disease. A number of such models approximating both spontaneous (IL-10, IL-2, TLR5, T-bet deficient mice) and induced (DSS, TNBS, Oxazolone) colitis have been established. Ever since the DSS-induced murine colitis model was developed by Okayasu, et al in 1990, it has been utilized in several thousand publications to evaluate a variety of genetically modified mice and test the efficacy of a number of therapeutics (Okayasu et al., 1990). By examining these models, several generalizations and conceptual ideas can be put forward.

In numerous models of spontaneous colitis, the gut microbiota is required and animals remain free from colitis if they are raised and maintained in germ-free conditions (Round and Mazmanian, 2009). Further, Helicobacter, a microbe whose presence varies from facility to facility, exerts a substantial influence over the development and severity of spontaneous or induced colitis and could thereby contribute to several discrepancies observed between models and laboratories. Another confounding constituent of the microbiota are segmented filamentous bacteria (SFB), a group which can induce IL-17+ $\mathrm{Th}_{17}$ cells. It has been shown that colonization of germfree mice with SFB-containing microbiota isolated from Taconic BL6 mice induced robust $\mathrm{Th}_{17}$ cell differentiation. However, colonization with a microbiota deficient in SFB, derived from BL6 mice obtained from Jackson Labs, resulted in only a modest increase in $\mathrm{Th}_{17}$ cells (Ivanov et al., 2009). 


\section{(i) The frenemy within: role of microbiota in DSS-induced colitis}

It has long been appreciated that proper immune development, nutrition and intestinal homeostasis requires a complete microbiota. An emerging concept in mucosal biology holds that proper interaction between the innate immune system, microbiota and associated ligands is a key determinant of the development and severity of DSS-colitis (Round and Mazmanian, 2009). It has been shown that depletion of microbiota by broad spectrum antibiotics exacerbates DSS-induced colitis (Rakoff-Nahoum et al., 2004). Interestingly, administration of ligands specific to TLRs 2 and 4, (lipoteichoic acid and LPS, respectively), along with DSS, provides substantial protection against DSS-induced colitis in microbiotadepleted WT mice (Rakoff-Nahoum et al., 2004). In addition, these TLR ligands did not protect against DSS-induced colitis in mice deficient in respective TLRs (Rakoff-Nahoum et al., 2004). Similarly, systemic administration of Poly (I:C), flagellin, CpG DNA, and muramyl dipeptide, respectively recognized by TLRs 3,5 and 9 and NOD2, provide significant protection against DSS-induced colitis (Rose et al., 2012; Vijay-Kumar et al., 2008; Vijay-Kumar et al., 2007; Watanabe et al., 2008). Further, the development of severe DSS-induced colitis is exacerbated in MyD88 deficient mice lacking all TLR signaling (with the exception of TLR3), including that required for production of the inflammasome cytokines IL-1 $\beta$ and IL-18 (Rakoff-Nahoum et al., 2004). This result further underscores the idea that the interaction between host innate immunity and gut microbiota is crucial in determining the outcome of DSS-induced gut pathogenesis. In models of induced intestinal inflammation, the microbiota is a double edged sword as it both protects against induction of chemical colitis while later driving the most damaging disease processes via dissemination of proinflammatory ligands.

\section{(ii) Gut epithelial apoptosis and proliferation: a balancing act in the gut dictates DSS- induced pathology}

The gastrointestinal tract is lined by a fragile monolayer of epithelial cells characterized by high turnover. The maintenance of intestinal epithelial barrier function in the intestine requires a delicate and dynamic balance between epithelial loss by apoptosis and the generation of new cells by proliferation. When this balance shifts towards either excessive or insufficient apoptosis, a broad range of gastrointestinal diseases can manifest. During DSS colitis, the turnover of epithelial cells is influenced by number of factors including diet, gut microbiota, several transcription factors and pro-inflammatory cytokines as well as interactions between these actors. For example, MyD88 deficient mice are highly susceptible to DSS-induced epithelial cell apoptosis and exhibit poor proliferation, aggravating disease (Rakoff-Nahoum et al., 2004).

The most potent pro-inflammatory cytokine, TNFa, is a major target of human IBD therapies and is highly upregulated during DSS-induced gut inflammation. TNFa signals via 2 receptors, TNFR1 and TNFRII. Signaling via TNFR1 is associated with apoptosis and cytokine production whereas signaling via TNFRII is associated with proliferation and enhanced cell survival. Interestingly, deletion of TNFRI aggravates not only DSS-induced colitis (Wang et al., 2012) but also TNBS colitis (Ebach et al., 2005), underscoring the importance of both apoptosis and proliferation in influencing the outcome of chemicallyinduced colitis.

\section{(iii) A matter of bias: strain-specific cytokine profiles influence DSS-induced colitis}

Cytokines produced by $\mathrm{T}$ helper cells $\left(\mathrm{Th}_{1}, \mathrm{Th}_{2}\right.$ and $\left.\mathrm{Th}_{17}\right)$ have an enormous impact on intestinal inflammation. The study of a number of animal models as well as human IBD indicates that $\mathrm{CD}$ is a process driven by $\mathrm{Th}_{1} / \mathrm{Th}_{17}$ cells (via production of IFN-g and IL-12) and that $\mathrm{UC}$ is driven by $\mathrm{Th}_{2}$ cells (via production of IL-4, IL-5, IL-10 and IL-13). DSSinduced colitis, while not a typical $\mathrm{Th}_{1}$-like model, more closely approximates human UC. It 
has been shown that, in DSS-induced chronic colitis, IL-4 secretion is upregulated and deletion of IL-4 greatly ameliorates disease severity (Stevceva et al., 2001). Yet, despite these shortcomings, DSS colitis offers the most reliable approximation of human disease currently available. It has been well established that IL-18 and IL-12 together strongly induce IFN- $\gamma$ (Dinarello, 1999). Interestingly, IL-18 in combination with IL-2 is a strong inducer of the $\mathrm{Th}_{2}$ cytokine IL-4 (Hoshino et al., 2000; Yoshimoto et al., 1999). Consistent with these observations, IL-12KO mice were significantly protected from DSS colitis whereas IL-18KO mice develop more severe disease (Takagi et al., 2003).

The DSS-colitis model has also shed light on the differences between mouse strains based on the relative progression of DSS-induced pathology. For example, the DSS colitis model was instrumental in establishing that C57BL6 and Balb/c mice are $\mathrm{Th}_{1}$ - and $\mathrm{Th}_{2}$-biased, respectively. Upon DSS challenge, both strains develop colitis accompanied by increased colonic levels of IL-1a/ $\beta$, IL-6, IL-18 and G-CSF. However, soon after DSS removal, Balb/c mice recovered to become free of symptoms within 2 weeks with complete recovery by 4 weeks as ascertained by histopathology and local cytokine production. C57BL/6 mice, on the other hand, continued to lose body weight after DSS removal and exhibited loose stools lasting as long as 5 weeks following cessation of DSS. Further, removal of an acute dose of DSS from C57BL/6 mice resulted in progression from acute to chronic inflammation with the manifestation of systemic symptoms. Because of the pleiotropic functions of cytokines, their influence on DSS-induced pathology can be rather complex with a full, in depth examination being beyond the scope of this chapter.

\section{(iv) Walking the high wire: the homeostatic role of NF-KB in intestinal inflammation}

$\mathrm{NF}-\kappa \mathrm{B}$ is a well characterized, ubiquitous transcription factor and primary mediator of the inflammatory response during inflammation. Recently, it has been demonstrated that NF- $\kappa B$ also mediates a potent cytoprotective, prosurvival homeostatic function under basal conditions in the gut. Accordingly, total deletion of IKK $\gamma$, an essential regulatory subunit of $\mathrm{NF}-\kappa \mathrm{B}$ in gut epithelial cells, results in spontaneous colitis in mice due to extensive epithelial apoptosis and loss of barrier function (Nenci et al., 2007). Similarly, pharmacological inhibition of IKK- $\beta$ results in pronounced DSS-induced inflammation (Eckmann et al., 2008). These IKK $\gamma$ deficient mice are rescued by deletion of MyD88, implying the importance of $\mathrm{NF}-\kappa \mathrm{B}$ signaling in preserving intestinal homeostasis. In addition to its homeostatic role under basal conditions, NF- $\kappa \mathrm{B}$ signaling plays a protective role during DSS-induced colitis despite this pathway's potential induction of proinflammatory cytokines. The loss of RelA, another critical component of NF- $\kappa \mathrm{B}$, exacerbates DSS-induced colitis in mice due to attenuated expression of anti-apoptotic genes (Steinbrecher et al., 2008). Thus, as with the presence and healthy composition of the microbiota, balanced and optimal epithelial NF-kB signaling is necessary to maintain intestinal homeostasis.

\section{(v). Leaving no stone unturned: Host dietary factors also influence gut inflammation}

Diet also plays a major role in determining susceptibility to IBD. Increased intake of total fat, total polyunsaturated fatty acids, omega- 6 fatty acids and meat were associated with an increased risk of UC (Hou et al., 2011). DSS has become instrumental in examining the underlying mechanisms by which high fat diets aggravate colitis (Ma et al., 2008; Teixeira et al., 2011; van der Logt et al., 2012). Another example is anemia resulting from iron deficiency, the most prevalent extra-intestinal complication of IBD as iron is a critically essential metabolic nutrient for both host and gut bacteria. The treatment of IBD-associated anemia involves oral, but not systemic, iron supplementation, as the latter can result in extensive adverse gastrointestinal side effects. Similarly, dietary, but not systemic, iron supplementation exacerbates DSS-induced colitis in mice, indicating a role for iron-induced 
oxidative stress, likely mediated by Fenton's reaction, during intestinal inflammation (Carrier et al., 2006; Seril et al., 2005). The iron axis represents just one example of a myriad known and unknown dietary factors that can influence disease progression directly and/or indirectly via effects on microbiota.

\section{CONCLUSION}

DSS colitis, despite it shortcomings, has been of great use in understanding the pathophysiology of intestinal inflammation and, consequently, has informed the theoretical and clinical appreciation of active IBD. This model, in skilled hands, represents a powerful tool with which to study the contribution of any aspect of the increasingly complicated gut environment or to evaluate interventions designed to prevent or ameliorate disease. The DSS-induced colitis model will continue to yield valuable mechanistic clues regarding the interactions between host genetics, gut innate immunity, microbiota, diet and other environmental factors in maintaining gastrointestinal homeostasis.

\section{Acknowledgments}

M.V-K was supported by NIH grants K01 (DK083275), R03 (094864), and R01 (DK097865), and BC is supported by the Crohn's and Colitis Foundation of America (CCFA) Research Fellowship Award.

\section{References}

Araki A, Kanai T, Ishikura T, Makita S, Uraushihara K, Iiyama R, Totsuka T, Takeda K, Akira S, Watanabe M. MyD88-deficient mice develop severe intestinal inflammation in dextran sodium sulfate colitis. Journal of gastroenterology. 2005; 40:16-23. [PubMed: 15692785]

Carrier JC, Aghdassi E, Jeejeebhoy K, Allard JP. Exacerbation of dextran sulfate sodium-induced colitis by dietary iron supplementation: role of NF-kappaB. International journal of colorectal disease. 2006; 21:381-387. [PubMed: 16133010]

Cerf-Bensussan N, Gaboriau-Routhiau V. The immune system and the gut microbiota: friends or foes? Nature reviews. Immunology. 2010; 10:735-744. [PubMed: 20865020]

Chassaing B, Darfeuille-Michaud A. The commensal microbiota and enteropathogens in the pathogenesis of inflammatory bowel diseases. Gastroenterology. 2011; 140:1720-1728. [PubMed: 21530738]

Chassaing B, Srinivasan G, Delgado MA, Young AN, Gewirtz AT, Vijay-Kumar M. Fecal lipocalin 2, a sensitive and broadly dynamic non-invasive biomarker for intestinal inflammation. PLoS One. 2012; 7:e44328. [PubMed: 22957064]

Dinarello CA. Interleukin-18. Methods. 1999; 19:121-132. [PubMed: 10525448]

Ebach DR, Newberry R, Stenson WF. Differential role of tumor necrosis factor receptors in TNBS colitis. Inflammatory bowel diseases. 2005; 11:533-540. [PubMed: 15905700]

Eckmann L, Nebelsiek T, Fingerle AA, Dann SM, Mages J, Lang R, Robine S, Kagnoff MF, Schmid RM, Karin M, Arkan MC, Greten FR. Opposing functions of IKKbeta during acute and chronic intestinal inflammation. Proceedings of the National Academy of Sciences of the United States of America. 2008; 105:15058-15063. [PubMed: 18815378]

Hoshino T, Yagita H, Ortaldo JR, Wiltrout RH, Young HA. In vivo administration of IL-18 can induce IgE production through Th2 cytokine induction and up-regulation of CD40 ligand (CD154) expression on CD4+ T cells. European journal of immunology. 2000; 30:1998-2006. [PubMed: 10940889]

Hou JK, Abraham B, El-Serag H. Dietary intake and risk of developing inflammatory bowel disease: a systematic review of the literature. The American journal of gastroenterology. 2011; 106:563-573. [PubMed: 21468064]

Ivanov II, Atarashi K, Manel N, Brodie EL, Shima T, Karaoz U, Wei D, Goldfarb KC, Santee CA, Lynch SV, Tanoue T, Imaoka A, Itoh K, Takeda K, Umesaki Y, Honda K, Littman DR. Induction 
of intestinal Th17 cells by segmented filamentous bacteria. Cell. 2009; 139:485-498. [PubMed: 19836068]

Johansson ME, Phillipson M, Petersson J, Velcich A, Holm L, Hansson GC. The inner of the two Muc2 mucin-dependent mucus layers in colon is devoid of bacteria. Proceedings of the National Academy of Sciences of the United States of America. 2008; 105:15064-15069. [PubMed: 18806221]

Kitajima S, Takuma S, Morimoto M. Histological analysis of murine colitis induced by dextran sulfate sodium of different molecular weights. Exp Anim. 2000; 49:9-15. [PubMed: 10803356]

Laroui H, Ingersoll SA, Liu HC, Baker MT, Ayyadurai S, Charania MA, Laroui F, Yan Y, Sitaraman SV, Merlin D. Dextran sodium sulfate (DSS) induces colitis in mice by forming nanolipocomplexes with medium-chain-length fatty acids in the colon. PLoS ONE. 2012; 7:e32084. [PubMed: 22427817]

Ma X, Torbenson M, Hamad AR, Soloski MJ, Li Z. High-fat diet modulates non-CD1d-restricted natural killer $\mathrm{T}$ cells and regulatory $\mathrm{T}$ cells in mouse colon and exacerbates experimental colitis. Clinical and experimental immunology. 2008; 151:130-138. [PubMed: 17991290]

Macdonald ML, Bissada N, Vallance BA, Hayden MR. Absence of stearoyl- CoA desaturase-1 does not promote DSS-induced acute colitis. Biochimica et biophysica acta. 2009; 1791:1166-1172. [PubMed: 19695343]

Nell S, Suerbaum S, Josenhans C. The impact of the microbiota on the pathogenesis of IBD: lessons from mouse infection models. Nature reviews. Microbiology. 2010; 8:564-577. [PubMed: 20622892]

Nenci A, Becker C, Wullaert A, Gareus R, van Loo G, Danese S, Huth M, Nikolaev A, Neufert C, Madison B, Gumucio D, Neurath MF, Pasparakis M. Epithelial NEMO links innate immunity to chronic intestinal inflammation. Nature. 2007; 446:557-561. [PubMed: 17361131]

Okayasu I, Hatakeyama S, Yamada M, Ohkusa T, Inagaki Y, Nakaya R. A novel method in the induction of reliable experimental acute and chronic ulcerative colitis in mice. Gastroenterology. 1990; 98:694-702. [PubMed: 1688816]

Poritz LS, Garver KI, Green C, Fitzpatrick L, Ruggiero F, Koltun WA. Loss of the tight junction protein ZO-1 in dextran sulfate sodium induced colitis. J Surg Res. 2007; 140:12-19. [PubMed: 17418867]

Rakoff-Nahoum S, Paglino J, Eslami-Varzaneh F, Edberg S, Medzhitov R. Recognition of commensal microflora by toll-like receptors is required for intestinal homeostasis. Cell. 2004; 118:229-241. [PubMed: 15260992]

Rose WA 2nd, Sakamoto K, Leifer CA. TLR9 is important for protection against intestinal damage and for intestinal repair. Sci Rep. 2012; 2:574. [PubMed: 22893852]

Round JL, Mazmanian SK. The gut microbiota shapes intestinal immune responses during health and disease. Nature reviews. Immunology. 2009; 9:313-323.

Seril DN, Liao J, Yang CS, Yang GY. Systemic iron supplementation replenishes iron stores without enhancing colon carcinogenesis in murine models of ulcerative colitis: comparison with ironenriched diet. Digestive diseases and sciences. 2005; 50:696-707. [PubMed: 15844705]

Steinbrecher KA, Harmel-Laws E, Sitcheran R, Baldwin AS. Loss of epithelial RelA results in deregulated intestinal proliferative/apoptotic homeostasis and susceptibility to inflammation. Journal of immunology. 2008; 180:2588-2599.

Stevceva L, Pavli P, Husband A, Ramsay A, Doe WF. Dextran sulphate sodium- induced colitis is ameliorated in interleukin 4 deficient mice. Genes and immunity. 2001; 2:309-316. [PubMed: 11607786]

Takagi H, Kanai T, Okazawa A, Kishi Y, Sato T, Takaishi H, Inoue N, Ogata H, Iwao Y, Hoshino K, Takeda K, Akira S, Watanabe M, Ishii H, Hibi T. Contrasting action of IL-12 and IL-18 in the development of dextran sodium sulphate colitis in mice. Scandinavian journal of gastroenterology. 2003; 38:837-844. [PubMed: 12940437]

Teixeira LG, Leonel AJ, Aguilar EC, Batista NV, Alves AC, Coimbra CC, Ferreira AV, de Faria AM, Cara DC, Alvarez Leite JI. The combination of high-fat diet-induced obesity and chronic ulcerative colitis reciprocally exacerbates adipose tissue and colon inflammation. Lipids Health Dis. 2011; 10:204. [PubMed: 22073943] 
van der Logt EM, Blokzijl T, van der Meer R, Faber KN, Dijkstra G. Westernized high-fat diet accelerates weight loss in dextran sulfate sodium-induced colitis in mice, which is further aggravated by supplementation of heme. J Nutr Biochem. 2012

Vijay-Kumar M, Aitken JD, Sanders CJ, Frias A, Sloane VM, Xu J, Neish AS, Rojas M, Gewirtz AT. Flagellin treatment protects against chemicals, bacteria, viruses, and radiation. Journal of immunology. 2008; 180:8280-8285.

Vijay-Kumar M, Wu H, Aitken J, Kolachala VL, Neish AS, Sitaraman SV, Gewirtz AT. Activation of toll-like receptor 3 protects against DSS-induced acute colitis. Inflammatory bowel diseases. 2007; 13:856-864. [PubMed: 17393379]

Wang K, Han G, Dou Y, Wang Y, Liu G, Wang R, Xiao H, Li X, Hou C, Shen B, Guo R, Li Y, Shi Y, Chen G. Opposite role of tumor necrosis factor receptors in dextran sulfate sodium-induced colitis in mice. PLoS ONE. 2012; 7:e52924. [PubMed: 23285227]

Watanabe T, Asano N, Murray PJ, Ozato K, Tailor P, Fuss IJ, Kitani A, Strober W. Muramyl dipeptide activation of nucleotide-binding oligomerization domain 2 protects mice from experimental colitis. The Journal of clinical investigation. 2008; 118:545-559. [PubMed: 18188453]

Xavier RJ, Podolsky DK. Unravelling the pathogenesis of inflammatory bowel disease. Nature. 2007; 448:427-434. [PubMed: 17653185]

Yan Y, Kolachala V, Dalmasso G, Nguyen H, Laroui H, Sitaraman SV, Merlin D. Temporal and spatial analysis of clinical and molecular parameters in dextran sodium sulfate induced colitis. PLoS ONE. 2009; 4:e6073. [PubMed: 19562033]

Yoshimoto T, Tsutsui H, Tominaga K, Hoshino K, Okamura H, Akira S, Paul WE, Nakanishi K. IL-18, although antiallergic when administered with IL-12, stimulates IL-4 and histamine release by basophils. Proceedings of the National Academy of Sciences of the United States of America. 1999; 96:13962-13966. [PubMed: 10570181] 
A

B

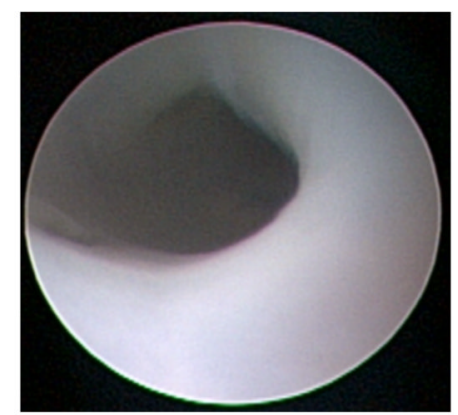

C Water only

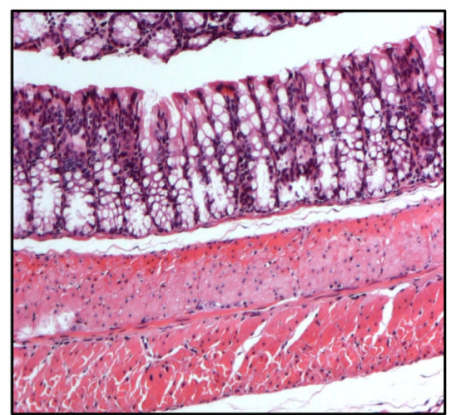

Water only

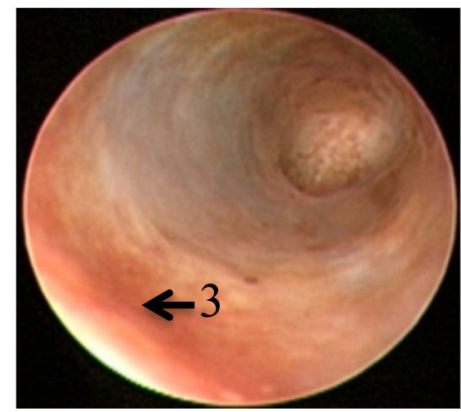

DSS

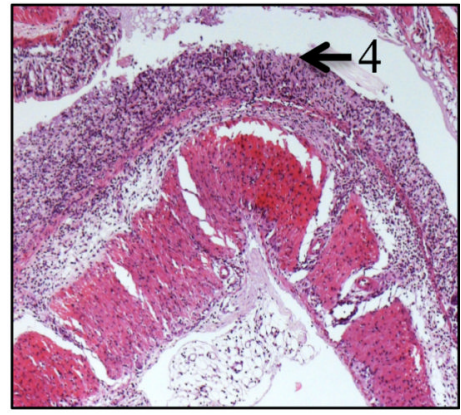

DSS

Figure 1.

Figure 1. DSS-induced intestinal inflammation in mice

Eight week old C57BL6 mice were given 2.5\% DSS in drinking water for 7 days. Control mice were given water only. On day 8 , mice were sacrificed and monitored for colitis. A. Gross picture of colons. B. Endoscopic examination of colons. C. H\&E stained colonic sections. Arrows indicate: 1. Shortened and bleeding colons, 2. Enlarged spleen, 3. Superficial inflammation, 4. Epithelial erosion and immune cell infiltration in DSS-treated mice. 


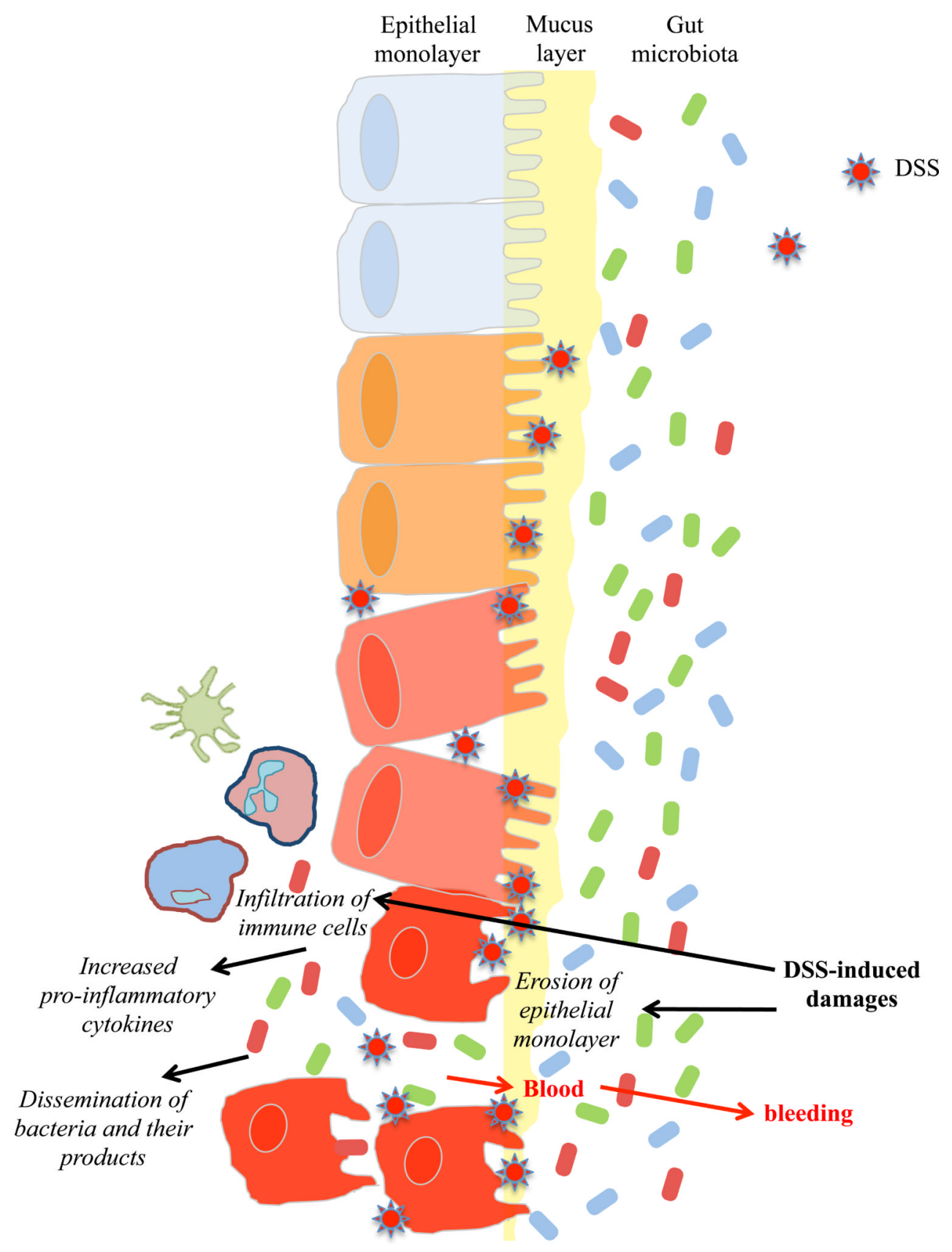

Figure 2

Figure 2.

Schematic representation of DSS-induced colitis. 